Trascender, Contabilidad y Gestión Núm. 8 (mayo - agosto del 2018).

ISSN: 2448-6388. Universidad de Sonora. Departamento de Contabilidad.

Reserva de Derechos 04-2015-04172070800-203.

\title{
Cumplimiento de la Armonización Contable en materia de transparencia en el Supremo Tribunal de Justicia del Estado de Sonora
}

Accounting harmonization in the matters of transparency in the supreme court of justice in the state of Sonora

$$
\text { Silvino Castellanos Trujillo }{ }^{1}
$$

\section{Resumen}

El tema de estudio a conocer es de la armonización contable en materia de transparencia; es un tema que con el paso del tiempo se ha ido implementado en algunos estados, municipios entidades y organismos; y a pesar del tiempo que se tiene de haberse creado esta Ley no se ha dado un avance significativo en el proceso de armonización e implementación de sistemas para operar y dar cumplimiento en base a la normatividad.

En esta investigación se busca obtener un conocimiento en materia de transparencia de los rubros que la conforman para generar un reporte detallado de la evaluación, control y seguimiento. La evaluación se lleva a cabo por el Instituto Superior de Auditoria y Fiscalización (ISAF) en conjunto con el Consejo Estatal de Armonización Contable (CEAC); en una plataforma informática denominada Sistema de Evaluación de Armonización Contable (SEVAC).

Esta investigación se realizó en uno de los tres poderes del ejecutivo, en esta caso en el Poder
Judicial del Estado de Sonora, integrado por el Supremo Tribunal de Justicia, seleccionado por formar parte de la prueba piloto y contar con información para el análisis para revisar su evaluación.

De la información analizada, se validó que en algunos apartados que integran el módulo de la transparencia presentan incumplimiento en el segundo, tercero y cuarto trimestre del 2017.

Esto concluye que aún los entes públicos no cuentan con una estructura o sistema adecuado para la armonización y desde luego el apego a la normatividad que marca la Ley General de Contabilidad Gubernamental (LGCG).

Palabras clave: Armonización Contable, Transparencia, Sonora.

DOI: https://doi.org/10.36791/tcg.v0i8.15

Recibido: 10 de abril de 2018.

Aceptado: 15 de junio de 2018.

\footnotetext{
${ }^{1}$ Silvino Castellanos Trujillo. Contador Público, Auditor del Instituto Superior de Auditoria y Fiscalización de Sonora (ISAF). Correo: silvino.castellanos@isaf.gob.mx.
} 


\section{Abstract}

The theme of this study is to know of the accounting harmonization in the matters of accounting related to transparency; is a theme that with the pass of time has been implementing in some states, cities, beings and organizations; yet with the time that has passed since the creation of this Law, there hasn't been a significative advance on the process for the harmonization and implementation of the systems to operate and give results to the law.

On this investigation we are looking to obtain the knowledge on the theme of transparency in the fields that build up to it as to make a detailed report about its evaluation, control and its development. The evaluation will be done by the ISAF (Instituto Superior de Auditoria y Fiscalizacion) together with the CEAC (Consejo Estatal de Armonizacion Contable) with the use of an informatic platform denominated SEVAC (Sistema de Evaluación de Armonización Contable).
On this investigation it will be around the three powers of the executive, in this case on the Power of the Attorney of the State of Sonora, which was selected to form part of the pilot test and count with information for the analysis and reviewing its results. From the information that was analyzed, it was validated that in some sections that form the transparency, they present problems keeping the objectives on the second, third and fourth trimester of 2017.

This concludes that the public entities are still lacking on the structure or the appropriate systems for the harmonization and the norms that places the LGCG (Ley General de Contabilidad Gubernamental).

Keywords: Accounting Harmonization, Transparency, Sonora. 


\section{Introducción}

La Armonización Contable hoy en día es un tema importante en el sector público, paulatinamente se ha ido implementado y estableciendo criterios uniformes para la generación y presentación de información financiera de los entes públicos en México con el objetivo de mostrar transparencia en sus operaciones.

El presente trabajo tiene la finalidad de aportar un conocimiento en el cumplimiento de los apartados que integran el módulo de transparencia en la Armonización Contable a través de un reporte detallado que conforman el módulo de transparencia y son: Pagos por conceptos de Ayudas Sociales, Información Contable, Información Presupuestaria, Información Programática e Inventarios.

Para lo cual se recabo información directa con los responsables de llevar a cabo este proceso de evaluación que es el ISAF y el CEAC. Es importante mencionar que las revisiones que hace el ISAF en sus auditoria es a través de un documento llamado Cuestionario de Cumplimiento de la Ley General de Contabilidad Gubernamental, como nuevas tecnologías incorporadas para la evaluación y seguimiento es a través de una plataforma informática denominada (SEVAC).

El SEVAC se define como:

"Herramienta informática desarrollada por la Secretaría Técnica del Consejo Nacional de Armonización Contable en términos del artículo 18 de las Reglas de Operación de los Consejos de Armonización Contable de las Entidades Federativas” (SEVAC, 2016 URL http://www.sevac.mx).

Esta plataforma fue creada por el Consejo Técnico del Consejo Nacional de Armonización Contable (CONAC) a partir del segundo semestre del 2016 y se puso en práctica el segundo semestre del 2017, como un prototipo a los tres Poderes de Gobierno Ejecutivo, Legislativo y Judicial, Organismos Autónomos y Municipios.
Actualmente en el primer semestre del 2018, esta herramienta informática incluyo a la entidades y dependencias para su evaluación. Los resultados obtenidos son importantes para el ISAF que realiza labores de fiscalización y en el caso de los Consejeros Estatales, porque son parte de la retroalimentación para conocer el avance en materia de la armonización contable en materia de transparencia de las entidades públicas que la conforman a nivel Estado.

\section{Antecedentes}

La Armonización Contable es un tema importante en el sector Público ya que con el tiempo se ha ido normalizando y estableciendo criterios uniformes para la generación de la información de los entes públicos que permiten el acercamiento de las normativas contables de los distintos países. Este es el objetivo básico de la armonización contable.

Entonces se puede decir que el concepto de Armonización Contables es:

“La armonización contable Internacional consiste en establecer unos criterios comunes que permitan el acercamiento de las normativas contables de los distintos" (Sanz Santoloria, 2003:98).

El proceso de armonización contable es deseable siempre y cuando los cambios o transformaciones sean graduales, asumiendo que con el cambio paulatinamente; también trae consecuencias que se tiene que ir atendiendo para poder cumplir el objetivo previsto. Hay organismos interesados en llevar a cabo una armonización contable de carácter público entre ellos se puede decir que son la Organización de Naciones Unidas (ONU) y la Organización para la Cooperación y el Desarrollo Económico (OCDE) que tiene participación a través de sus gobiernos.

La LGCG implica también crear un sistema que aporte información contable, financiera y presupuestal desde el momento mismo en que se planean los presupuestos y programas es decir que se registran a la par los 
momentos contables como presupuestales. También plantea registrar los derechos de cobro de los ingresos, así como su control y resguardo en el casos de bienes que conforman el patrimonio de la entidad (Clemente Sanchez, 2010).

La armonización también ha tenido sus inicios de carácter privado de la contabilidad y auditoría que son; La Comisión de Normas Internacionales de Contabilidad (IASC) y la Federación Internacional de Contadores (IFAC) (Sanz Santoloria, 2003).

El día 28 de octubre 2003 en el Palacio Nacional se llevó a cabo una convención en donde asistieron varios funcionarios para dar a conocer una serie de propuestas relacionadas con la contabilidad gubernamental; en donde mediante una propuesta de reforma integral de la Secretaria de Hacienda Pública incorporan un elemento innovador el cual viene a mejorar la transparencia denominado “Armonización Contable Nacional” (Sanz Santoloria, 2003).

Una vez dado a conocer este tema, se crea la reforma Ley General de Contabilidad Gubernamental que emana del artículo 73 fracción XXVIII de la Constitución Política de los Estados Unidos Mexicanos (CPEUM), la cual fue publicada el día 7 de mayo del 2008, y puesta en práctica en enero 2009. Su objetivo general de esta Ley es establecer criterios generales para la contabilidad gubernamental y así como la emisión de la información financiera (Camara de Diputados de $\mathrm{H}$ Congreso de la Unión, 2016).

La Ley General de Contabilidad Gubernamental está compuesta un Consejo Nacional de Armonización Contable denominado (CONAC) según artículo 6 de la LGCG, el cual tiene como funciones, emitir normas y lineamientos para la generación de información financiera aplicada a los entes.

El CONAC está conformado por un comité técnico denominado Secretaría Técnica del Consejo Nacional de Armonización. Este Consejo apegándose al artículo 18 de las Reglas de Operación de los Consejeros Armonización Contables de Entidades Federativas, el cual señala la creación de una Plataforma informática en apoyo a la evaluación de la Armonización Contable (CONAC, 2016).

La idea de involucrarse y entrar en detalle en el tema de la Armonización Contable es conocer su normatividad, su aplicación en materia de cumplimiento y lo importante de que existan organismos fiscalizadores con ciertos colabores Nacionales y Estatales, para dar seguimiento a la evaluación de los apartados de Registros Contables, Registros Presupuestarios, Administrativos, Cuenta Pública y Otros Cuestionarios (Inventarios) que conforman la LGCG y por supuesto como parte clave del proceso, la transparencia en donde recae la responsabilidad de publicar y transparentar todos los movimientos y operaciones de los entes públicos, el cual implica tener una mejor rendición de cuentas.

Por lo que se procederá a investigar el cumplimiento de la armonización contable en materia de transparencia en el Supremo Tribunal de Justicia del ejercicio 2017, para obtener un reporte detallado de los apartados que integran el módulo de transparencia, ya que este organismo ha formado parte de una prueba piloto de la evaluación en la plataforma del SEVAC durante los últimos tres trimestres del 2017, y primer trimestre del 2018, a través del ISAF, que es el organismo fiscalizador responsable de validar los resultados obtenidos de los entes públicos con la colaboración del CEAC.

\section{Justificación}

La armonización contable hoy en la actualidad es un tema de suma importancia para el sector público; como una medida de realizar una mejor transparencia de la información contable de las entidades públicas e implementar sistemas con criterios comunes; para generar información contable y que sea comparable entre las demás entidades públicas. 
Por ende, a nivel nacional la Armonización Contable trata de conseguir uniformidad en los métodos y prácticas contables. A nivel internacional buscar establecer criterios que sean comunes y que permitan un acercamiento en las normas contables de los países (Sanz Santoloria, 2003).

El concepto de transparencia se puede decir que es un principio democrático que consiste en mostrar los asuntos públicos; la práctica de colocar está información en la "vitrina pública". Es evidente poner las acciones gubernamentales y el ejercicio de los presupuestos públicos a la vista de todos lo cual constituye una condición primordial para la rendición de cuentas y el combate a la corrupción; de ahí la trascendencia de su evolución y fortalecimiento. Es importante mencionar que la transparencia no implica un acto de rendir cuentas a un destinatario especifico, sino colocar la intermediación de estas "vitrinas públicas" (Ugalde, 2002).

Por ende la importancia de la transparencia juega un papel muy importante en la Armonización Contable porque se puede decir que a través de ella se publican los resultados, operaciones e información financiera, por lo que es muy importante validar que se esté cumpliendo con la publicación de la información de manera trimestral y en los formatos de Evaluación Trimestral Contabilidad Armonizada, ETCA y la Cuenta Pública Contabilidad Armonizada (CPCA) establecidos por la Secretaria de Hacienda del Estado de Sonora. En estos formatos se publican la información Contable, Presupuestal y Programática de los entes públicos, como objetivo de la armonización de unificar criterios en la presentación de la información contable.

Aunado a esta situación se seleccionó este tema a manera de Investigación, para tener un conocimiento en la evaluación en materia de transparencia, el cual permita al lector a la sociedad y a las personas involucradas en el sector público, tener información al respecto para conozcan que si se está cumpliendo con la normatividad que marca la Ley General de Contabilidad Gubernamental.

Los beneficios que se pretenden comprobar con esta investigación es la de conocer el grado de avance en el control, evaluación y seguimiento en la armonización contable en materia de transparencia con la colaboración con entidad de Fiscalización Superior Local que sería el ISAF y los organismos a nivel Nacional CONAC y estatal CEAC.

Para llevar a cabo está investigación se establece un objetivo general el de Conocer el cumplimiento de la Armonización Contable, en materia de transparencia en el Supremo Tribunal de Justicia del estado de Sonora, con el fin de presentar un reporte detallado de los apartados que integran el módulo de transparencia, para ello tenemos que revisar los siguientes puntos:

1.- Analizar la normatividad de la LGCG en el ámbito del Supremo Tribunal de Justicia del Estado de Sonora.

2.- Identificar los elementos que componen la plataforma del SEVAC.

3.- Revisar el módulo de transparencia en la información de Publicación de Ayudas y Subsidios, Contable, Presupuestaría, Programática e Inventarios.

4.- Revisar los resultados publicados del ente público en el módulo de transparencia para validar si cumplen con la normatividad de la Ley General de Contabilidad Gubernamental.

El marco teórico en el cual se fundamenta la investigación será en lo jurídico apegado al marco legal que marca la normatividad de la presente ley para su estudio considerada para la presente investigación. La normatividad emana de la reforma del artículo 73 de la Constitución Política de los Estados Unidos Mexicanos en la fracción XXVIII. 
El ámbito de aplicación de la presente Ley es para los tres poderes de gobierno (Poder Ejecutivo, Legislativo y Judicial, los estados y el Distrito Federal; los ayuntamientos de los municipios; los órganos políticoadministrativos de las demarcaciones territoriales del Distrito Federal; las entidades de la administración pública paraestatal ya sean federales, estatales o municipales y los órganos autónomos federales y estatales (Camara de Diputados de H Congreso de la Unión, 2016).

A partir del año 2009 entro en vigor esta Ley para que todos los entes públicos a nivel nacional se apeguen a la presente Ley. Cabe aclarar que durante este periodo esta Ley ha sufrido varias reformas; la primera se dio el 12 de noviembre de 2012, el 9 de diciembre de 2013, el 30 de diciembre de 2015 y la última reforma fue el día 27 de abril de 2016 (Sanz Santoloria, 2003).

En el artículo 6 de la Ley General de Contabilidad Gubernamental contempla la creación de un consejo que se le denomina Consejo Nacional de la Armonización Contable (CONAC) que tiene por objeto la emisión de normas y lineamientos para la generación de información financiera de los entes públicos. A la fecha este consejo ha emitido más de 80 acuerdos normativos en los distintos rubros como en normas de patrimonio, ingresos, egreso, para la presentación de información, difusión de información gubernamental y reglas de registros como normas del desempeño (Camara de Diputados del H Congreso de la Union, 2016).

Los siguientes apartados son lo que se revisan durante el proceso de evaluación en plataforma.

Publicación del Título V: En este apartado se compone de un formato en el cual se publican todo con relación a los montos pagados por el concepto de ayudas y subsidios que proporciona el ente público.

Información contable: Esta información se compone de 9 formatos mediante los cuales el ente público plasma la información y se publican en la página de transparencia y son:
Estado de Actividades: documento que muestra la situación actual en cuestión monetaria,

Estado de Situación Financiera: documento que muestra la utilidad o pérdida del ente público.

Estado de variación de Hacienda Pública: Te revela el flujo de recursos recibidos y ejercidos

Estado de cambios en la Situación Financiera: Te ayuda a revisar el origen y destino de los recursos.

Flujo de efectivo: Te mide el flujo en cuestión monetaria.

Informe de Pasivos Contingentes: Se publica información de posibles pasivos como contingencia.

Informe de las Notas Financieras: Se publica todas las notas con respecto al Estado de la Situación Financiera y Estado de Actividades.

Estado Analítico del Activo: Su finalidad es mostrar el comportamiento de los fondos, valores, derechos y bienes debidamente identificados y cuantificados en términos monetarios, que dispone el ente público para realizar sus actividades.

Estado Analítico del Deuda y otros Pasivos: Su finalidad es mostrar las obligaciones insolutas de los entes públicos, al inicio y fin de cada período, derivadas del endeudamiento interno y externo.

Información Presupuestaria: Esta información se compone de 7 formatos mediante los cuales el ente público genera la información y se publica en la página de transparencia y son:

Estado Analítico de Ingresos: Se analizan los ingresos a nivel detalle del ente público.

Estado Analítico de egresos con clasificación administrativa: Se revisan los egresos cuando un ente tiene diferentes unidades administrativas.

Estado Analítico de Egresos con clasificación económica del gasto: Se revisa el egreso, pero por tipo de gasto. 
Estado Analítico del ejercicio de presupuesto de egresos por objeto de gasto: Se revisa el egreso a nivel capítulo y cuentas que integran el capítulo.

Estado Analítico del ejercicio de presupuesto por clasificación funcional: Se revisa la finalidad y función del egreso.

Endeudamiento Neto: Publicación de la deuda si hay.

Intereses de la deuda: Se publican los intereses si hay.

Información Programática: Esta información se compone de 3 formatos mediante los cuales el ente público genera la información y se pública en la página de transparencia y son:

Publicación del gasto por categoría de programa: Se revisa que el ente publique el gasto por categoría y de acuerdo con el programa que se estableció.

Publicación de la MIR Matriz de Indicadores de Resultados: Se revisa que la MIR este publicada y autorizada por la Secretaria de Hacienda.

Publicación de los programas proyectos de inversión: Se revisa que se publiquen los proyectos si hay.

Publicación de Inventarios: En este apartado se compone de un formato en el cual se evalúa la publicación de los inventarios de acuerdo con lo que marca Ley; que se debe de hacer inventarios 2 veces en el año.

Nota: Este apartado solo se evalúa en el segundo y cuarto trimestre de acuerdo a lo que marca la LGCG.

La presente investigación se llevará a cabo en el estado de Sonora. La entidad pública que será objeto de investigación forma parte de los tres poderes del Ejecutivo, Legislativo y Judicial en este caso se seleccionó al Poder Judicial del Estado el cual se constituye por el Supremo Tribunal de Justicia ubicado en el Boulevard Rio Sonora y calle Comonfort sin número en el tercer piso en el Edifico Hermosillo localizado en la Oficinas de Gobierno del vado de rio.

\section{Metodología}

El método usado en la presente investigación será inductivo porque va de lo particular a lo general y el alcance de la investigación será descriptiva; es decir un estudio a detalle de los procesos y profundización en la información de los datos que se recopilaron durante el proceso.

Durante la investigación se presentan dos patrones que son objeto de estudio, el primero en donde intervienen dos variables que fue el ente público Supremo Tribunal de Justicia como independiente y dependiente la Ley de Transparencia ya que este último depende del Supremo de Tribunal de Justicia de lo que valla a contestar con respecto a la normatividad que le aplique.

En el segundo panorama intervienen una variable independiente que es la Ley General de Contabilidad Gubernamental y dos variables dependientes que depende de la Ley; en este caso el ente público porque se apega a lo que marca la Ley y en el caso del sistema de la plataforma SEVAC, también se apega a esta Ley porque es evaluado bajo el marco legal de la normatividad de la LGCG. Los resultados de estas variables serán cuantitativos que pueden ser medidas numéricamente.

La población que se investigó fue uno de los tres poderes del Ejecutivo, en este caso es el Poder Judicial del Estado, el cual se delimito a investigar al Supremo Tribunal de Justicia, ubicado en la localidad de Hermosillo, Sonora, México.

Este ente público fue seleccionado porque es uno de los tres poderes, que participa en la prueba prototipo, para la evaluación del seguimiento en materia de armonización contable en la plataforma del SEVAC.

A continuación, en la siguiente figura se presentan los organismos que intervienen en la evaluación del sistema SEVAC. Figura 1 Organismos que interviene en la evaluación de la Plataforma SEVAC. 
Figura 1. Organismos que intervienen en la evaluación de la Plataforma SEVAC

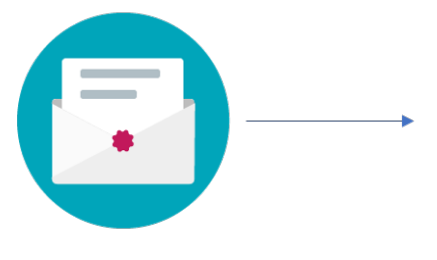

CONAC

(Consejo Nacional de Armonización Contable) Emite Reglas de Operación

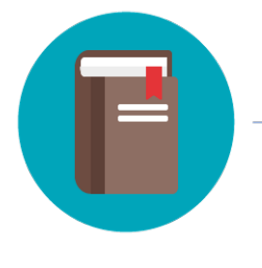

ASF

(Auditoría Superior de la Federación) Emite marco metodológico

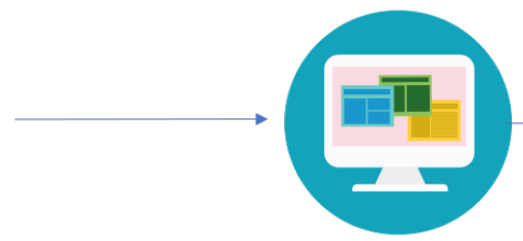

EFSL

(Entidades de Fiscalización Superior Locales)

Validan los resultados de sus entidades

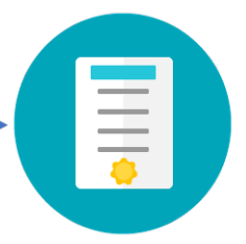

Consejos Estatales

Informan avances de su entidad

Fuente: SEVAC página URL http://www.sevac.mx

En la siguiente figura se explica brevemente como funciona la evaluación en el sistema de la plataforma.

Figura 2. Funcionamiento General del SEVAC

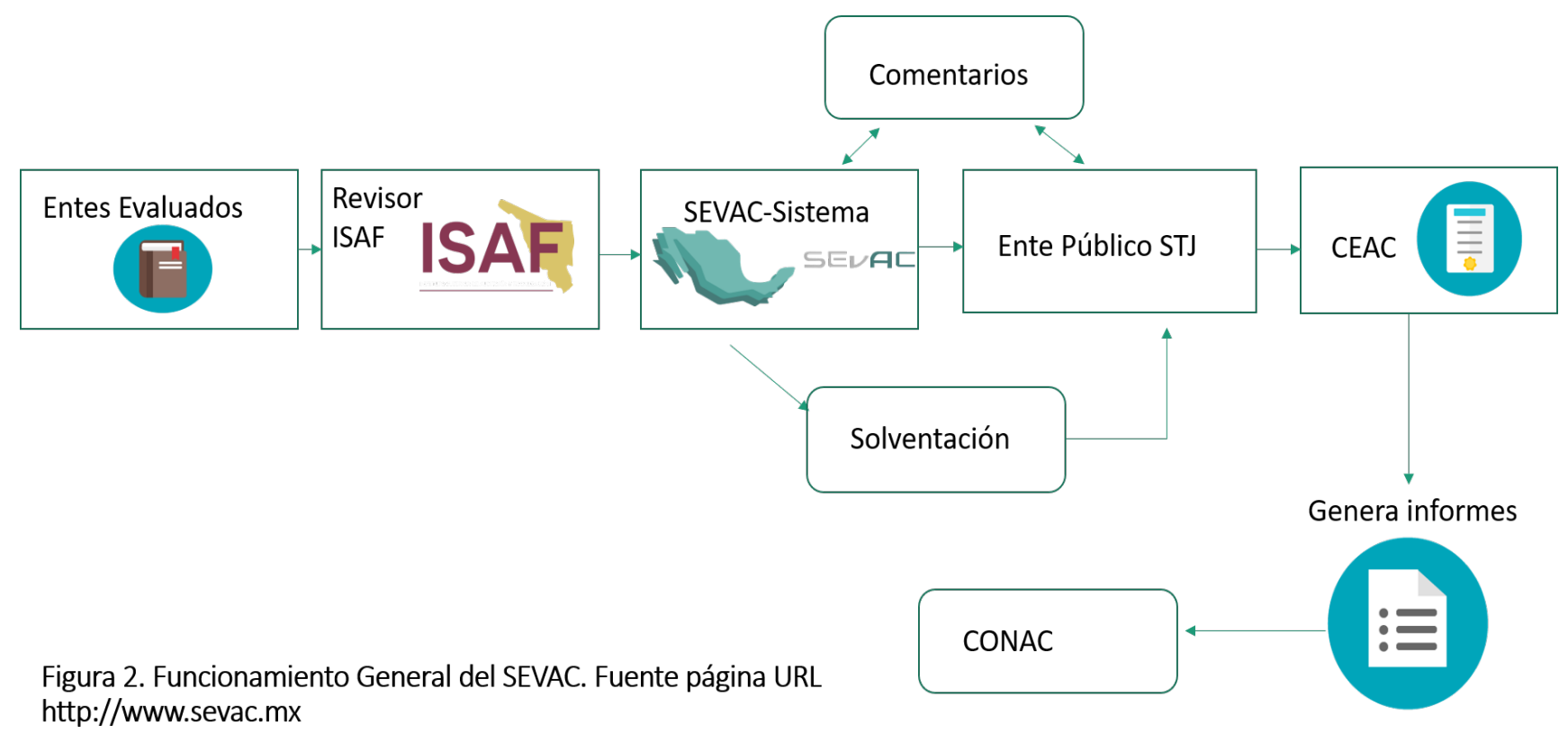

Figura 2. Funcionamiento General del SEVAC. Fuente página URL http://www.sevac.mx 


\section{Análisis de Información}

Información del segundo trimestre 2017, el contenido Contable tuvo un cumplimiento del 89\%, ya que no se publicó el Estado de Actividades según Figura 1.

Figura 3. Evaluación 2 Trimestre 2017

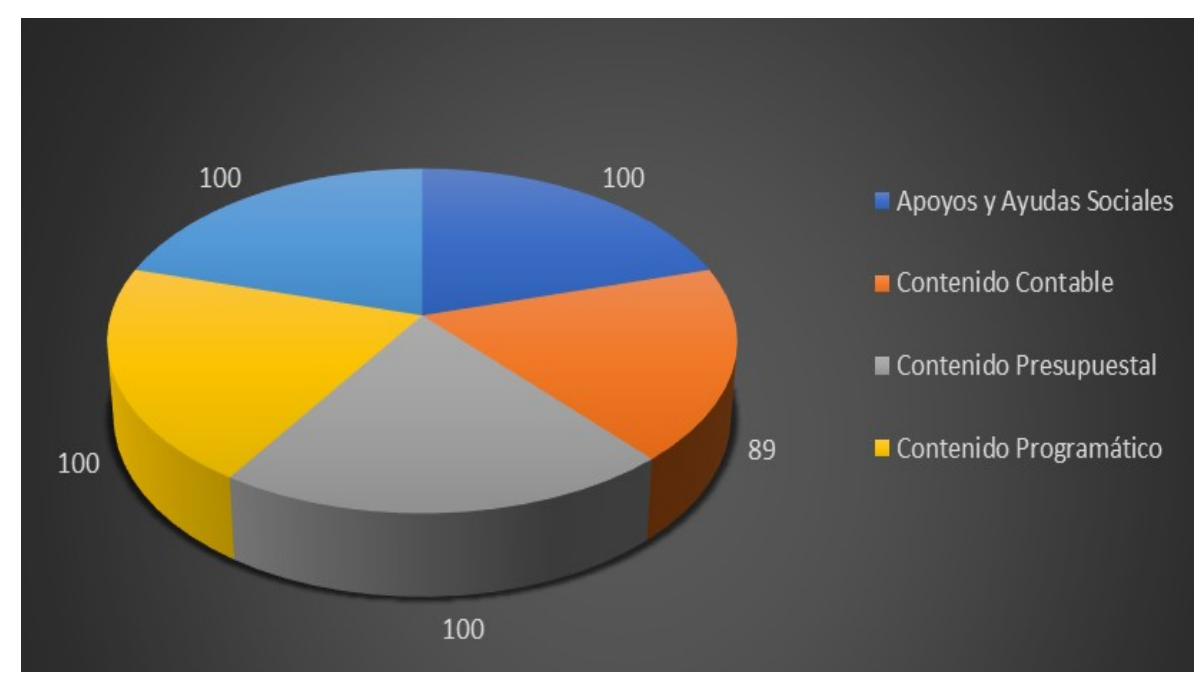

Fuente: Evaluación SEVAC 2017, pp. 1 y 3.

Información del tercer trimestre 2017, el contenido Presupuestal tuvo un cumplimiento del 97\% porque no público el Estado Analítico de Ingresos y en el contenido Programático tuvo un cumplimiento 67\% , porque no presento la Matriz de Indicadores de Resultados (MIR) según Figura 2.

Figura 4. Evaluación 3 Trimestre 2017

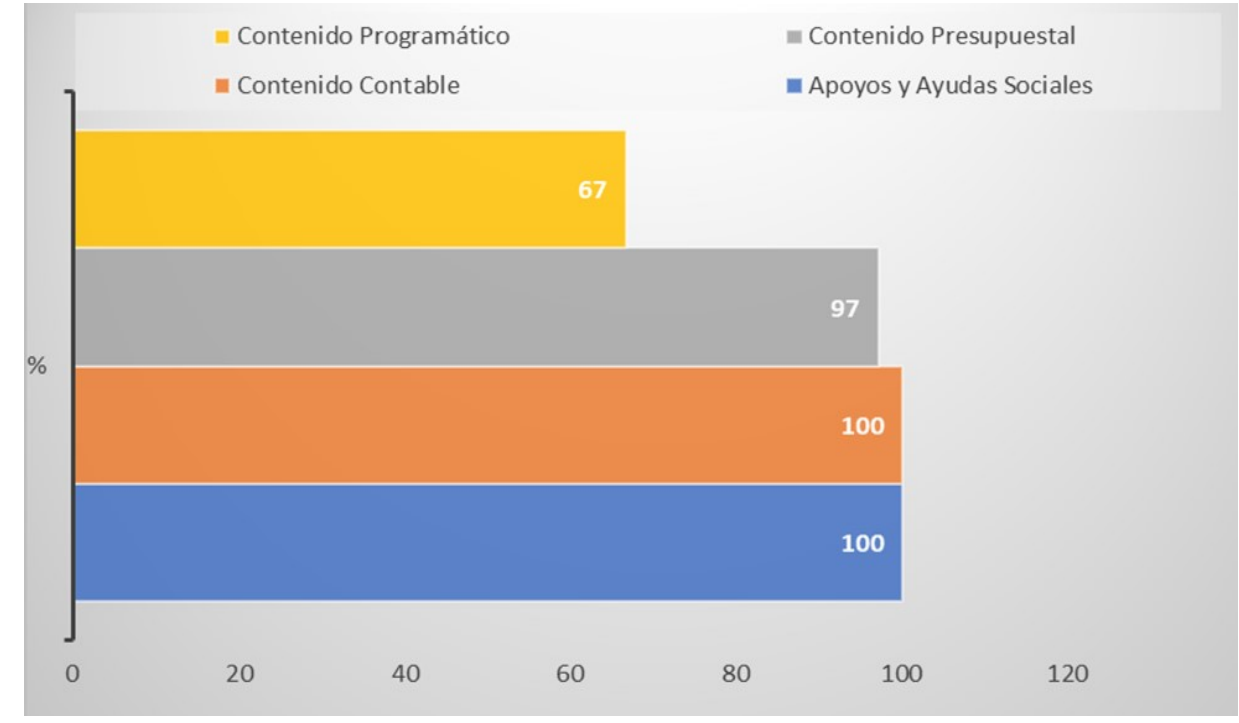

Fuente: Evaluación SEVAC 2017, p. 2. 
Información del cuarto trimestre 2017, el contenido Programático tuvo un cumplimiento del 87\%, ya que no público la Matriz de Indicadores de Resultados y en el contenido de Otras publicaciones se tuvo un cumplimiento del $60 \%$ porque no público el inventario de bienes muebles e inmuebles según Figura 3.

Figura 5. Evaluación Inventario 3 trimestre 2017

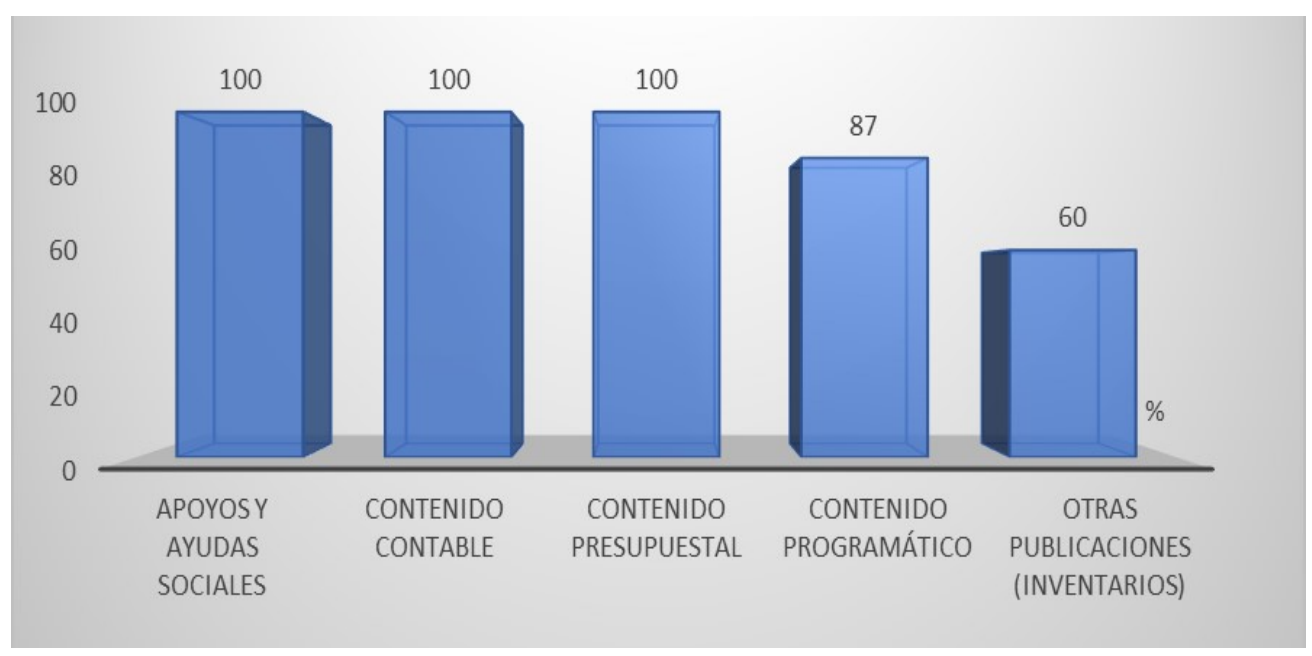

Fuente: Evaluación SEVAC 2017.

\section{Conclusiones}

Como parte de la investigación se analizó la información de los apartados de publicación por concepto de Ayudas Sociales, Contenido Contable, Contenido Presupuestal, Contenido Programático y Publicación de Inventarios, que conforman el módulo de transparencia del Supremo Tribunal de Justicia.

Después de revisar el reporte detallado por cada apartado; se llegó a la conclusión de que no están cumpliendo con lo que marca la normatividad de LGCG, en materia de Transparencia.

Esto debido a que no tenían la información completa y armonizada en algunos apartados que contempla la transparencia que son los contables, presupuestal, programático e inventarios aparte que el sistema que opera el Supremo Tribunal de Justicia (SAP) no es un sistema adecuado y viable para lo que contempla la LGCG; ya que se adaptó a los lineamientos para que pueda operar bajo los criterios que implica esta Ley.
De igual manera, se encontró que existen documentos como el Estado de Actividades que no presentaron, Analítica de ingresos, la Matriz de indicadores que en dos semestres no presentan la información por desconocer de la normatividad, así como los inventarios a comentarios del responsable del área de Contabilidad del Supremo Tribunal de Justicia no tiene los inventarios actualizados, porque debido a los cambios del sistema de justicia se han incrementado las unidades, por lo que a veces es imposible hacer todo un levantamiento en todas la regiones, por falta de personal, sistemas y recursos, por lo que no fue posible cumplir con el apartado de transparencia.

Este problema se viene agudizando debido a la falta de presupuesto para operar que es la base para iniciar, se hizo una reforma pero no se contempló estos factores presupuesto, sistemas, capacitación y tecnología que implican un gasto para operar; por lo que se debió haber presupuestado porque hay entidades y municipios que no cuentan con algunos de estos factores que son esenciales para poner en marcha la reforma. 
Por último, es importante señalar que se debe de considerar el seguimiento a la reforma para ir midiendo el avance del cumplimiento en materia de armonización contable, no solo basta en crear leyes, reformas y decretos implica un proceso planeación en considerar varios factores como presupuestar sistemas, tecnología, capital humano y capacitación para implementar y llevar a cabo una reforma de este tipo.

\section{Referencias}

Camara de Diputados de H Congreso de la Unión. (2016). Constitución Politica de los Estados Unidos Mexicanos. Mexico (págs. 63-67). Mexico: Diario Oficial de la Federación.

Camara de Diputados del H Congreso de la Union. (2016). Ley General de Contabilidad Gubernamental. México (págs. 1-5). México: Diario Oficial de la Federación.
Clemente Sanchez, E. (2010). La Armonización Contable esta en Ruta. Politica Digital, 56, 42-43.

CONAC. (2016). Reglas de Operación de los Consejeros de Armonización Contable de las Entidades Federativas. México (pág. 4). México: Diario Oficial de la Federación.

Sanz Santoloria, C. J. (2003). Armonización Contable Internacional de caracter Profesional : El IASB. España: Universidad Zaragoza.

SEVAC. (29 de 02 de 2017). Sistema de Evaluación y Armonización Contable. Obtenido de Sistema de Evaluación y Armonización Contable: http://www.sevac.mx

Ugalde, L. C. (2002). Rendición de Cuentas y Democracia, El caso de México. México: Instituto Federal Electoral. 